\title{
BMJ Open How to measure cultural competence when evaluating patient-centred care: a scoping review
}

\author{
Sadia Ahmed, ${ }^{1}$ Fartoon M Siad, ${ }^{2}$ Kimberly Manalili, ${ }^{2}$ Diane L Lorenzetti, ${ }^{2}$ \\ Tiffany Barbosa, ${ }^{3}$ Vic Lantion, ${ }^{3}$ Mingshan Lu, ${ }^{4}$ Hude Quan, ${ }^{2}$ Maria-Jose Santana ${ }^{1,2}$
}

To cite: Ahmed S, Siad FM, Manalili $\mathrm{K}$, et al. How to measure cultural competence when evaluating patient-centred care: a scoping review. BMJ Open 2018;8:e21525. doi:10.1136/ bmjopen-2018-021525

- Prepublication history and additional material for this paper are available online. To view these files, please visit the journal online (http://dx.doi. org/10.1136/bmjopen-2018021525).

Received 5 January 2018 Revised 18 May 2018

Accepted 25 June 2018

D Check for updates

(c) Author(s) (or their employer(s)) 2018. Re-use permitted under CC BY-NC. No commercial re-use. See rights and permissions. Published by BMJ.

${ }^{1}$ Department of Paediatrics, Cumming School of Medicine, University of Calgary, Calgary, Alberta, Canada

${ }^{2}$ Department of Community Health Sciences, University of Calgary, Calgary, Alberta, Canada

${ }^{3}$ Ethno-Cultural Council of Calgary, Calgary, Alberta,

Canada

${ }^{4}$ Department of Economics, University of Calgary, Calgary, Alberta, Canada

Correspondence to

Sadia Ahmed;

sadahmed@ucalgary.ca

\section{ABSTRACT}

Objectives The purpose of this study was to identify patient-centred quality indicators (PC-QI) and measures for measuring cultural competence in healthcare.

Design Scoping review.

Setting All care settings.

Search strategy A search of CINAHL, EMBASE, MEDLINE, PsycINF0, Social Work Abstracts and SocINDEX, and the grey literature was conducted to identify relevant studies. Studies were included if they reported indicators or measures for cultural competence. We differentiated PC-Qls from measures: PC-Qls were identified as a unit of measurement of the performance of the healthcare system, which reflects what matters to patients and families, and to any individual that is in contact with healthcare services. In contrast, measures evaluate delivery of patient-centred care, in the form of a survey and/or checklist. Data collected included publication year and type, country, ethnocultural groups and mention of quality indicator and/or measures for cultural competence. Results The search yielded a total of 786 abstracts and sources, of which 16 were included in the review. Twelve out of 16 sources reported measures for cultural competence, for a total of 10 measures. Identified domains from the measures included: physical environment, staff awareness of attitudes and values, diversity training and communication. Two out of 16 sources reported PCQls for cultural competence (92 structure and process indicators, and 48 outcome indicators). There was greater representation of structure and process indicators and measures for cultural competence, compared with outcome indicators.

Conclusion Monitoring and evaluating patientcentred care for ethnocultural communities allows for improvements to be made in the delivery of culturally competent healthcare. Future research should include development of PC-Qls for measuring cultural competence that also reflect cultural humility, and the involvement of ethnocultural communities in the development and implementation of these indicators.

\section{INTRODUCTION}

Racial and ethnic minorities in Western countries experience greater adverse health outcomes, such as higher rates of cardiovascular disease, cancer and diabetes, ${ }^{1}$ and tend to receive a lower quality of care. ${ }^{23} \mathrm{It}$

\section{Strengths and limitations of this study}

- Transparent and rigorous search strategy.

- Involvement of community partners in the study.

- Updated peer-reviewed and grey literature search in 2017.

- Search strategy using only English terms.

- We did not assess the quality of the measures and/ or indicators identified.

is recognised that patient-centred care (PCC) can help improve health outcomes and is a key dimension of high-quality care. ${ }^{4} \mathrm{PCC}$ is a model of care involving patients and families in the planning, development and assessment of their care. ${ }^{5}$ A PCC model has been associated with improved interactions between healthcare providers and their patients. ${ }^{6-8}$ PCC benefits patients and families and healthcare systems. For instance, decreases in healthcare utilisations such as fewer diagnostic tests and referrals, ${ }^{9}$ decreasing patient's length of stay in hospital and ultimately improving the efficiency and cost of care. ${ }^{10-12}$ In addition, PCC that is culturally competent aims to reduce disparities in health and healthcare. ${ }^{13}$ The National Quality Forum defines culturally competent care as the 'ongoing capacity of healthcare systems, organizations, and professionals to provide for diverse patient populations high-quality care that is safe, patient and family-centred, evidence based, and equitable. ${ }^{14}$ PCC that is culturally competent is necessary to meet the healthcare needs of diverse populations. ${ }^{15}$

With a growing population of immigrants and refugees in Canada and other Western countries, there is an increasing need to meet their healthcare needs. For instance, Canada welcomed 33723 Syrian refugees between November 2015 and November 2016. ${ }^{16}$ In response to a survey on accessing health services, $49 \%$ of Syrian refugees reported unmet healthcare needs, with access to care 
(time to see a healthcare provider, long wait times) and cost of care being the main issues. ${ }^{16}$ Various ethnocultural groups, especially those who are immigrants and refugees, experience a number of barriers to accessing quality care such as language and informational barriers, which can impact efforts to deliver PCC. ${ }^{17}{ }^{18}$ Lack of communication between patients and healthcare providers leads to distrust in both the provider and healthcare system, which can affect care-seeking behaviour. ${ }^{2}$ Limited levels of culture-related knowledge, skills, and experience and awareness of healthcare providers have been found to contribute to patients' low adherence to treatment. ${ }^{19}$ Culturally competent care aims to address these barriers and different expectations of care. ${ }^{20}$ Further, evidence has shown that greater cultural competence has been associated with improved doctor communication and better patient experiences. ${ }^{21}$ 'Cultural humility' proposed by Tervalon and Murray-García provides a different perspective to cultural competence, by emphasising reflection of one's self and acknowledging existing power imbalances between provider and patient. ${ }^{22}$ Cultural humility encompasses flexibility, acceptance of differences and willingness to learn from others as cultural informants. ${ }^{23}$ Cultural safety, another reflective concept similar to cultural humility, involves recognition of the range of cultural influences on an individual including but not limited to ethnicity, gender, age, sexual orientation, lifestyle choices, beliefs and values. ${ }^{24}$

Measures such as surveys and checklists are ways to assess delivery of PCC. These measures can be administered to patients, healthcare providers and healthcare staff to assess domains relating to the delivery of PCC and cultural competence. Domains are categories assigned to items within a measure (such as communication and patient-centred decision-making). However, despite efforts to develop measures for cultural competence, there have been criticisms about the way some measures assess cultural competence. Among these criticisms include: a static view of culture, assumptions that knowledge and confidence are enough to provide culturally competent care and the lack of measures incorporating cultural humility. ${ }^{25}{ }^{26}$ These criticisms and the complexity of measuring cultural competence have hindered measurement efforts.

Quality indicators (QI) are key metrics for evaluating healthcare quality that can determine whether improvements for care have been made, and allows for monitoring and longitudinal evaluation of care. ${ }^{27} \mathrm{~A}$ general definition provided by the Agency for Healthcare Research \& Quality (AHRQ) defines QIs as, 'standardized, evidence-based measures of health care quality that can be used with readily available hospital inpatient administrative data to measure and track clinical performance and outcomes. ${ }^{28}{ }^{29}$ However, quality of care is a difficult concept to measure as it is shaped by values of the society and goals of the current healthcare system. ${ }^{30}$ Different jurisdictions may have different priorities for the provision of quality care. ${ }^{30}$ Developing a standard set of indicators based on what matters to patients and families allows for standardised measurement across jurisdictions and healthcare facilities.

To ensure that the provision of care is truly patient centred and culturally competent, there is a need for development of PCC QIs to measure cultural competence. Without indicators, it is difficult to monitor whether improvements are being made in the delivery of healthcare. The objective of this scoping review is to identify existing patient-centred quality indicators (PC-QI) and measures for measuring cultural competence in healthcare. The identification of QIs and measures for cultural competence will contribute to the development and evaluation of a standard set of QIs for PCC implemented across healthcare settings.

\section{METHODS}

The Arksey and O'Malley's scoping review method$\operatorname{ology}^{31}$ and Levac et al's framework ${ }^{32}$ were used to guide the scoping review. The six stages in undertaking the scoping review were: (1) identifying the research question; (2) identifying relevant studies; (3) selecting studies; (4) charting the data; (5) collating, summarising and reporting the results; and (6) consulting with relevant stakeholders. A scoping review methodology was used, as the goal of the study was to gain an overview of the research into PC-QIs and measures for measuring cultural competence. We searched the published peer-reviewed and grey literature for cultural competence measures and indicators in PCC that have been developed and/or implemented across various point-of-care settings.

\section{Stage 1: identifying the research question}

Through consultation with the research team, the research questions for the search were developed. The search was guided by the questions: 'Are there cultural competence indicators for measuring patient-centred care?' and 'Have PC-QI's for cultural competence been implemented and evaluated across various care settings?'

In defining a QI, we adapted the AHRQ definition to incorporate the patient perspective as a unit of measurement of the performance of the healthcare system, which reflects what matters to patients and families, and to any individual that is in contact with healthcare services. This working definition provided a guide for the identification of QIs. In contrast, measures evaluate delivery of PCC, in the form of a survey and/or checklist, and they can inform the development of PC-QIs.

\section{Stage 2: identifying relevant studies}

Data sources and search strategy

The following electronic databases were searched from 1 January 1990 to 31 December 2016: CINAHL, EMBASE, MEDLINE, PsycINFO, Social Work Abstracts and SocINDEX, using search terms in English developed in consultation with our research librarian (DLL) such as 'patient-centred care', 'cultural competence', 
'ethno-cultural communities' and 'quality indicators' (24 January 2017) (online supplementary file 1). A researcher and community partner both searched the unpublished grey literature using the 'Google' search engine and similar search terms (15 June 2017). The reference lists of included studies in English were also scanned to identify any other studies of additional relevance from 1 January 1990 to 31 December 2016.

\section{Citation management}

All references were imported into a custom-written Java software application, Synthesis for improved reference management and data collection. ${ }^{33}$ Duplicate citations were removed automatically by the software, with any mismatched duplicates removed manually if detected.

\section{Stage 3: study selection and data abstraction}

Studies were included in the review if they reported on PCC indicators or measures for cultural competence, as identified by the author of the publications. Our search was limited to published peer-reviewed and grey literature in English from Canada, New Zealand, Australia, the UK and the USA (countries with greater number of immigrant populations). Data collected included publication year and type, country, ethnocultural groups and mention of QI or measures for cultural competence.

Two reviewers (MJS, ML) independently screened each identified title and abstract for eligibility. The updated peer-reviewed search was conducted by two additional reviewers (FMS, KM) to capture all sources up to December 2016, and the grey literature search was conducted by two other reviewers $(\mathrm{SA}, \mathrm{TB})$ to capture all sources up to June 2017. Any abstract selected for inclusion by either or both reviewers was then retrieved for full-text review. The reference lists of eligible full-text papers were hand-searched to identify additional papers of relevance to this review. Disagreements at any stage were discussed between the two reviewers and resolved through discussion with a third investigator and reached consensus.

\section{Stages 4 and 5: data collection and classification of measures and indicators}

A data collection tool was developed and adapted from a previous study ${ }^{34}$ to include characteristics specific to this review (online supplementary file 2). Extracted study characteristics included country; year of publication; type of study/article; ethnocultural, racial and/or diverse groups; indicators; and measures. Additionally, domains from all the measures were extracted.

A scoping review allows for main concepts from a research area to be mapped and provides an idea of what evidence is available for that research area. ${ }^{31}$ The goal of the review is to provide an overview of the research, rather than assessing the quality of individual studies. For this review, once QIs and measures were extracted, domains identified from the measures were classified according to the Donabedian model of quality of care assessment as a way to summarise the findings..$^{35}$ The model categorises healthcare quality into the categories of 'structure,' 'process' and 'outcome'. This conceptual model was used to classify the domains from the measures, as it is a widely recognised and adopted model to evaluate healthcare quality. ${ }^{30}$ Additionally, the person-centred care framework, ${ }^{36}$ developed by our research team, was used to classify the indicators identified from the review into person-centred care domains. The domains identified from the measures and from the person-centred care framework will be compared to identify domains specific to culturally competent care that can be used to inform the development of PCC indicators for measuring cultural competence.

\section{Stage 6: consultation with stakeholders Patient and public involvement}

Levac et al recommend that consultation with stakeholders should be an essential component of the scoping study methodology. ${ }^{32}$ The involvement of community partners allows for suggestions of additional references as well as the provision of insights beyond those in the literature. ${ }^{32}$ Partnerships with communities have been outlined as a strategy by the WHO for improving PCC. ${ }^{37}$ For this scoping review particularly, we included two community partners in the research team to consult on the research questions, aid in the search strategy and provide input on the grey literature search.

\section{RESULTS}

A total of 786 studies were identified through database and grey literature searching. Of these, 196 fulltext sources were assessed for eligibility; and 16 met the criteria for inclusion in this scoping review (peer reviewed $=5$, grey literature $=11$ ) (figure 1 ). Twelve out of 16 sources reported measures for cultural competence, for a total of 10 measures. Identified domains from the measures included: physical environment, staff awareness of attitudes and values, diversity training and communication. Two out of 16 sources reported PC-QIs for cultural competence (92 structure and process indicators, and 48 outcome indicators).

\section{Description of articles and types of cultural competence measures}

Table 1 presents the characteristics of the articles and grey literature sources included in the review. The years of publication of articles ranged from 1998 to 2017, and countries of publication were Canada $(n=6)$, USA $(n=9)$ and Australia $(n=1)$. Sources were original research articles $(n=5)$, guidelines/manuals $(n=4)$, toolkits $(n=3)$, reports $(n=2)$, a government document $(n=1)$ and a thesis $(n=1)$.

Twelve sources included measures for culture competence, for a total of 10 measures. Of these, five were provider self-assessments, such as 'Self-Assessment Tool for Primary Health Care Professionals' and 


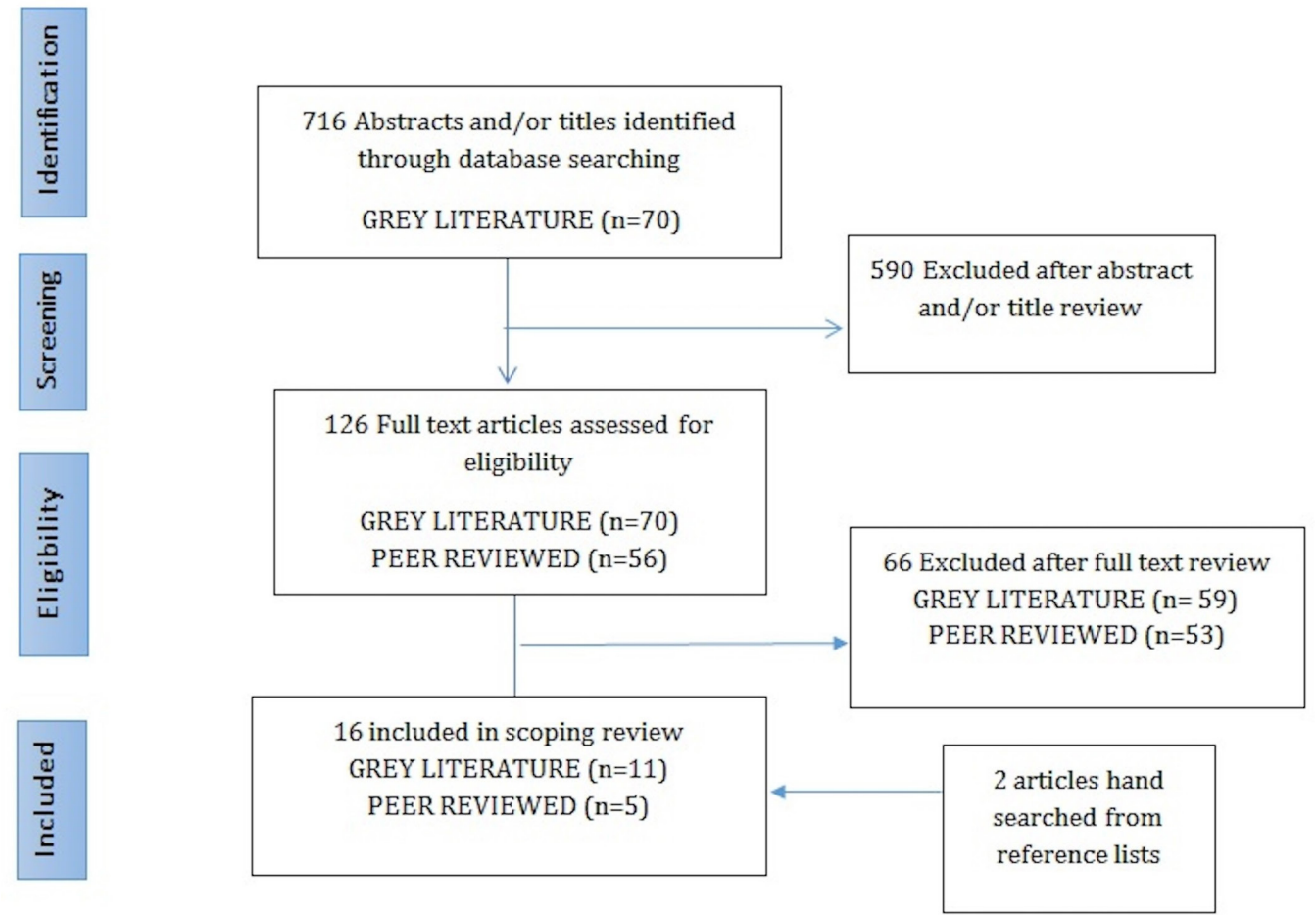

Figure 1 Preferred Reporting Items for Systematic Reviews and Meta-Analyses (PRISMA) flow diagram of article selection.

'Diversity Awareness Self-Reflection tool'. Two measures, the Consumer Assessment of Healthcare Providers and Systems (CAHPS) Cultural Competence (CC) Item Set, ${ }^{38}$ and the Interpersonal Processes of Care Survey, ${ }^{39}$ were specifically developed to assess patients' perspectives of their care. The remaining three were organisational assessment tools: the Cultural Competency Assessment Tool for Hospitals (CCATH) ${ }^{40}$ the Organizational Assessment

\section{Table 1 Characteristics for included sources $(n=16)$}

\begin{tabular}{lc}
\hline Country & $\begin{array}{c}\text { Number (\%) of 16 } \\
\text { sources }\end{array}$ \\
\hline Canada & $6(37.5)$ \\
\hline USA & $9(56.3)$ \\
\hline Australia & $1(6.3)$ \\
Year of publication & \\
\hline $2010-2017$ & $5(31.3)$ \\
\hline $2000-2009$ & $10(62.5)$ \\
\hline 1990-1999 & $1(6.3)$ \\
Document type & \\
\hline Original research (peer reviewed) & $5(31.3)$ \\
\hline Guideline/manual & $4(25)$ \\
\hline Toolkit & $3(18.8)$ \\
\hline Report & $2(12.5)$ \\
\hline Government document & $1(6.3)$ \\
\hline Thesis & $1(6.3)$ \\
\hline Mention of quality indicator & $2(12.5)$ \\
\hline
\end{tabular}

for Diversity and Cultural Competence, ${ }^{41}$ and Workplace Assessment Tool: Successful Practice Guidelines. ${ }^{42}$

Using the Donabedian quality of care assessment, cultural competence domains identified from the measures were categorised into structure, process and outcome..$^{35}$ There was a greater representation of measures assessing the processes and structures of care, than outcomes of care (table 2). Structure domains identified from the measures such as the physical environment, organisational policies and procedures for diversity and inclusiveness, organisational composition and climate, values and attitudes of staff, and workforce training and skills were specific to assessing cultural competence. Process domains identified from the measures assessing cultural competence were communication, shared decision-making, access to interpreter services, equitable treatment and trust.

\section{Description of the cultural competence PC-Qls}

Two sources (out of 16) identified cultural competence indicators to measure PCC (for a total of 92 structure and process indicators, and 48 outcome indicators), and we classified them using the person-centred care framework. ${ }^{36}$ Table 3 presents examples of the indicators identified from the two sources. The Lewin Group report presented structure indicators that aim to create a PCC culture and provide a supportive and accommodating environment. ${ }^{43}$ Examples included: 'policies, protocols regarding client/family/community input' and 'policy in place that minimizes the use of family members as interpreters' ${ }^{43}$ The Lewin Group report also presented guidelines to support the development of culturally 
Table 2 Domains identified from measures assessing cultural competence

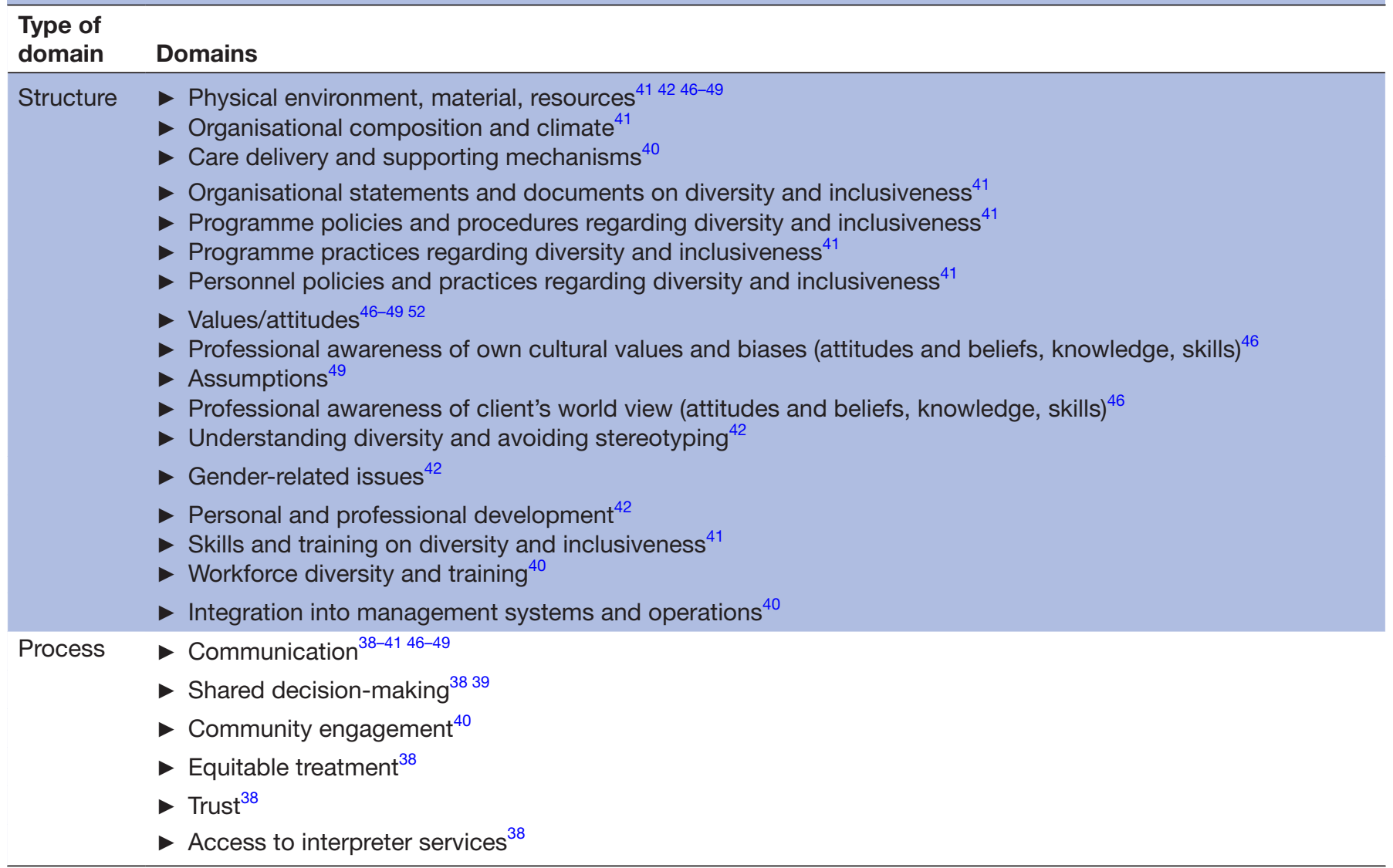

competent healthcare systems, but they were labelled as 'indicators' by the authors of the report. An example of a guideline from the report: 'Provides for staff training on use of interpreters'. ${ }^{43}$ The authors of the Lewin Group report defined indicators as 'particular observable or measurable characteristics of an organization that signify cultural competence ${ }^{43}{ }^{4}$ The National Standards for Culturally and Linguistically Appropriate Services, a review of the published and unpublished literature, and an expert panel of advisors that shared insights, information and

Table 3 Examples of indicators for cultural competence ${ }^{\star}$, classified according to the person-centred care framework ${ }^{36}$

\begin{tabular}{|c|c|c|}
\hline Type of indicator & Domains & Example indicators \\
\hline \multirow[t]{2}{*}{ Structure indicators } & Creating a PCC culture & $\begin{array}{l}\text { Policies, protocols regarding client/family/community } \\
\text { input }{ }^{43} \\
\text { System for informing patients of right to free } \\
\text { interpretation/translation services }{ }^{43} \\
\text { Percentage and retention of community members on } \\
\text { governing body and advisory committees } \\
\text { Formal cultural competence-related policies exist } \\
\text { regarding: personnel recruitment/retention, training/staff } \\
\text { development, language access/communication, cultural } \\
\text { competence-related grievances/complaints, community/ } \\
\text { client input }{ }^{43}\end{array}$ \\
\hline & $\begin{array}{l}\text { Providing a supportive and } \\
\text { accommodating environment }\end{array}$ & $\begin{array}{l}\text { Policy in place that minimises the use of family } \\
\text { members as interpreters }\end{array}$ \\
\hline
\end{tabular}

${ }^{*}$ Almost all indicators (except for one) were identified from the Lewin Group report: Indicators of Cultural Competence in Health Care Delivery Organizations: An Organizational Cultural Competence Assessment Profile. ${ }^{43}$

PCC, patient-centred care. 
opinions informed the development of indicators in the report. ${ }^{43}$ To gain perspectives on the utility and feasibility of the cultural competence indicators, the project team also visited various healthcare delivery sites. ${ }^{43}$ Evaluation and implementation of the indicators were mentioned as future directions. $^{43}$

The Strengthening Primary Care Access report presented one potential process indicator: per cent of immigrants, vulnerable women, Aboriginal, and so on, receiving Pap test/colorectal and breast cancer screening in a culturally appropriate manner. ${ }^{44}$ This indicator is presented as a potential indicator for primary care that is 'culturally safe'. ${ }^{44}$ Derived from Health Quality Ontario's access-related indicators, this indicator was designed to measure the experiences of immigrant populations, vulnerable women and Aboriginal women. ${ }^{44}$ The authors of the report suggest that community-based participatory research frameworks supporting collaborations with community members can enhance the development of appropriate 'culturally safe' indicators. ${ }^{44}$ Evaluation of indicators for primary care and implementation were not discussed in the document.

\section{DISCUSSION}

This review identified a vast body of research on cultural competence in PCC. While there is a number of measures for assessing cultural competence in the form of surveys and checklists, from the sources identified in the scoping review, standardised measures used for assessing cultural competence are lacking. Additionally, there is no current, standard set of PCC QIs for assessing cultural competence from the sources identified. This review is the first to examine PCC QIs for assessing cultural competence in healthcare. Measuring cultural competence and PCC through indicators is necessary to ensure that patients are receiving quality care that is sensitive to their healthcare needs. The identified measures found in this review provide the potential foundation for the development of indicators to assess cultural competence in PCC.

In order to effectively and systematically measure cultural competence in PCC, indicators should be developed and implemented. Most indicators identified were in the form of a policy to guide structural changes in care or guidelines to assess the organisation. Identified domains from measures were found to be similar in concept to the person-centred care domains, such as communication, creating a PCG culture and providing a supportive and accommodating environment. The domains identified from the measures, and domains from the person-centred care framework can help set priorities for the development of cultural competence indicators for monitoring and evaluating PCC.

Indicators should be presented as percentages or proportions, to guide monitoring of healthcare quality. Health quality organisations such as the AHRQ, Health Quality Ontario and other quality improvement agencies use percentages or proportions as the unit of measurement for quality improvement. ${ }^{28}$ This unit of measurement allows for comparison across facilities and facilitates longitudinal evaluation, measuring care that is truly culturally competent, and patient centred. Indicators are often derived from measures. Measures such as the CAHPS CG Item $\mathrm{Set}^{38}$ and the $\mathrm{CCATH}^{40}$ are validated measures, and are potential data sources for the development of indicators.

Most indicators found in the review were structure and process indicators. The presence of structure indicators for cultural competence is vital in building the foundation for process and outcome indicators. ${ }^{36}{ }^{43}$ Both the Lewin Group document and the person-centred care framework have outlined the importance of structure indicators. ${ }^{3643}$ Structure refers to the necessary materials, healthcare resources and organisational characteristics that are the foundation of PCC. ${ }^{36}$ Domains such as the care environment, supporting a diverse workforce and access to interpreter services, as well as using surveys and data sources such as the CCATH and self-assessments can inform the development of structure indicators. Other aspects of care include processes of care which refer to the interaction between patient and healthcare providers. ${ }^{36}$ Development of process indicators that are culturally competent can be informed by the process domains found in our review, including: communication, trusting relationship and equitable treatment. The development of outcome indicators such as patient-reported outcomes and patient-reported experiences is vital in determining the effects of care on the health status of patients and populations, and also in how patients perceive the care received. ${ }^{36}$ From the sources identified, there was also no mention of access to care in relation to distance, a domain captured under 'outcomes of care' from the person-centred care framework as a QI or a domain. ${ }^{36}$

This review found a lack of evidence on the evaluation and implementation of indicators in care settings. The National Quality Forum provides criteria for the evaluation of indicators, including: importance, scientific acceptability, feasibility, and usability and use. ${ }^{45}$ The authors of the Lewin Group report visited healthcare delivery sites to gain perspectives on the utility and feasibility of the indicators for cultural competence. ${ }^{43}$ Indicators that are developed should be evaluated according to set criteria, such as the one provided by the National Quality Forum, in order to ensure the indicators are validated and can be used across health facilities. Evaluation of indicators also allows for implementation of indications in the care settings for which they are developed.

Only one source identified in the review reported a measure for cultural competence that incorporated aspects of 'cultural humility' and cultural safety in the development. ${ }^{46}$ The Multicultural Practices Competencies tool incorporated the clinicians' self-reflection of race, ethnicity and power as well as an understanding of the factors (oppression, racism, and so on) impacting the health of minority patients. ${ }^{46}$ Many of the measures included domains in the delivery of culturally competent 
care such as communication and attitudes of healthcare professionals towards patients. ${ }^{41} 46-49$ However, cultural competence needs to go beyond these domains to include self-reflection and critique. Cultural humility can be attained through community-based partnerships, which was mentioned in the two sources with indicators. ${ }^{43} 44$ The process of self-reflection also includes an examination of power imbalances between patient and provider. ${ }^{13}$ In alignment with PCC, cultural humility involves a partnership between the patient and provider. ${ }^{22}$ A cultural humility and safety perspective shifts our view of culture, and can prevent stereotyping of people of diverse backgrounds, especially in the delivery of healthcare. ${ }^{13}$ In order for development of measures and indicators that reflect cultural humility and safety, there is a need for collaboration with patients, families and other community members. Specifically, collaboration with ethnocultural communities ensures various perspectives of quality of care are incorporated. Various sources mentioned working with patients and community partners in the development of priorities for care..$^{43} 44^{50}$ A study by Fongwa $e t$ al provides a good example of the inclusion of patients in understanding quality of care, as they interviewed African-American, Latino and White patients to gain an understanding of what matters to them in their healthcare.$^{51}$ Resulting themes from patient and community input can guide the development of indicators. Community-based partnerships are key to the codesign of indicators, and ensure care is patient centred.

\section{Strengths and limitations}

Our methodology and search strategy is transparent and rigorous, ensuring researchers have all the tools necessary to conduct the same search. Throughout the research processes, all records of the searches were kept, and a data abstraction form was used. This review was supported by our research team with expertise in knowledge synthesis and scoping reviews. As a first step in developing QIs for cultural competence, we collaborated with two community members. The development of a partnership between researchers and community patients is a first step to achieving PCC. Our community partners were included in our research team to consult on the research topic and questions, aid in an unbiased search strategy and provide input on the manuscript development.

This scoping review did not assess the quality of the studies and sources identified, therefore extraction of measures from strong and weak studies is considered. Our search results may also be biased, despite involvement of a community partner (TB), as we conducted the search using only terms in English, leading to our sources being in English.

\section{CONCLUSIONS}

This scoping review is the first to identify PCC QIs for measuring cultural competence in the literature. Monitoring and evaluating PCC for ethnocultural communities allows for improvements to be made in the delivery of culturally competent care. The identification of PCC measures and indicators for cultural competence in this scoping review is a key first step in laying the groundwork for developing evidence-based PC-QIs for cultural competence. Future research should include development of indicators for cultural competence in PCC, involvement of ethnocultural communities in the development and consensus of indicators and development of measures and indicators reflecting cultural humility.

Acknowledgements We acknowledge our community partners, the Ethno-Cultural Council of Calgary, who have provided tremendous support for our patient-centred care research with ethnocultural communities. We especially thank the Executive Director, Ms Marichu Antonio, as well as our Community Connectors/Research Assistants, including: Stephen Li, Ruba Samaan, Hala Said, Arlene Beradio, Aneesh Joseph and volunteers: Catherine Enstas and Bhim Tiwari. Additionally, we acknowledge the contributions of Ms Lisa Goodman and Ms Rachel Jolley, who provided support for the grey literature search for this scoping review. We also thank the Alberta Strategy for Patient Oriented Research Support Unit, as well as our research Patient-Partner, Ms Sandra Zelinsky for her ongoing contributions to the design and dissemination of this research.

Contributors MJS conceived the study, and SA, FMS, KM, ML, MJS, DLL, TB and VL identified key literature to be included in the review. MJS and ML independently screened each identified title and abstract for eligibility. The updated peer-reviewed search was conducted by FMS and KM, and the grey literature search was conducted by SA and TB. DLL provided guidance on the search strategy. SA led the drafting of the manuscript, design of the tables (with feedback from all authors) and management of references. MJS, FMS, KM, ML, DLL, TB and VL provided input on various drafts of the manuscript. All authors provided important intellectual contribution and guidance throughout the development of the manuscript. $\mathrm{HQ}, \mathrm{KM}$, $\mathrm{DLL}$ and $\mathrm{ML}$ provided guidance on the presentation of the findings and guidance on final revisions. All of the authors (SA, FMS, KM, DLL, TB, VL, ML, HQ, MJS) contributed to critical review and revisions to the manuscript, agreeing on the final version.

Funding MJS received funding from the MSI Foundation (Grant No 886) based in Alberta, Canada. ML acknowledges support from the Alberta's Strategy for Patient Oriented Research (SPOR). KM acknowledges support from the Ward of the 21st Century, University of Calgary.

Disclaimer All authors had access to the data (literature identified and tables) in the study and can take responsibility for the integrity of the data and the accuracy of the data analysis. The lead author affirms that this manuscript is an honest, accurate and transparent account of the study being reported; that no important aspects of the study have been omitted; and that any discrepancies from the study as planned (and, if relevant, registered) have been explained.

Competing interests None declared.

Patient consent None declared.

Provenance and peer review Not commissioned; externally peer reviewed.

Data sharing statement № additional data available.

Open access This is an open access article distributed in accordance with the Creative Commons Attribution Non Commercial (CC BY-NC 4.0) license, which permits others to distribute, remix, adapt, build upon this work non-commercially, and license their derivative works on different terms, provided the original work is properly cited, appropriate credit is given, any changes made indicated, and the use is non-commercial. See: http://creativecommons.org/licenses/by-nc/4.0/.

\section{REFERENCES}

1. Baldwin DM. Disparities in health and health care: focusing efforts to eliminate unequal burdens. Online J Issues Nurs 2003;8:2.

2. Institute of Medicine. Smedley $B D$, Stith $A Y, N e l s o n A R$, et al. Unequal treatment: confronting racial and ethnic disparities in health care. Washington: National Academy Press, 2003.

3. Asch SM, Kerr EA, Keesey J, et al. Who is at greatest risk for receiving poor-quality health care? N Engl J Med 2006;354:1147-56. 
4. Institute of Medicine. Crossing the quality chasm: a new health system for the 21st century. National Academy Press 2001.

5. Simces Z. ARCHIVED - Exploring the Link Between Public Involvement/ Citizen Engagement and Quality Health Care: A Review and Analysis of the Current Literature. 2005 https://www.canada.ca/en/healthcanada/services/health-care-system/reports-publications/quality-care/ exploring-link-between-public-involvement-citizen-engagementquality-health-care.html?=undefined (accessed 01 Oct 2017).

6. Teutsch C. Patient-doctor communication. Med Clin North Am 2003;87:1115-45.

7. Laidsaar-Powell RC, Butow PN, Bu S, et al. Physician-patientcompanion communication and decision-making: a systematic review of triadic medical consultations. Patient Educ Couns 2013;91:3-13.

8. Kim S, Brock DM, Hess BJ, et al. The feasibility of a multi-format Web-based assessment of physicians' communication skills. Patient Educ Couns 2011;84:359-67.

9. Oates J, Weston WW, Jordan J. The impact of patient-centered care on outcomes. Fam Pract 2000;49:796-804.

10. McMillan SS, Kendall E, Sav A, et al. Patient-centered approaches to health care: a systematic review of randomized controlled trials. Med Care Res Rev 2013;70:567-96.

11. Mead N, Bower P. Patient-centred consultations and outcomes in primary care: a review of the literature. Patient Educ Couns 2002;48:51-61.

12. Ayanian JZ, Markel H. Donabedian's Lasting Framework for Health Care Quality. N Engl J Med 2016;375:205-7.

13. Beach MC, Saha S, Cooper LA. The role and relationship of cultural competence and patient-centeredness in health care quality: Commonwealth Fund. New York, NY 2006.

14. Forum NQ. A Comprehensive Framework and and Preferred Practices for Measuring and Reporting Cultural Competency: A Consensus Report. Washington DC: National Quality Forum, 2008.

15. Betancourt JR, Green AR, Carrillo JE, et al. Defining cultural competence: a practical framework for addressing racial/ ethnic disparities in health and health care. Public Health Rep 2003;118:293-302.

16. Oda A, Tuck A, Agic B, et al. Health care needs and use of health care services among newly arrived Syrian refugees: a cross-sectional study. CMAJ Open 2017;5:E354-E358.

17. McKeary M, Newbold B. Barriers to Care: The Challenges for Canadian Refugees and their Health Care Providers. J Refug Stud 2010;23:523-45.

18. Bulman KH, McCourt C. Somali refugee women's experiences of maternity care in west London: A case study. Crit Public Health 2002;12:365-80.

19. Shapiro J, Hollingshead J, Morrison EH. Primary care resident, faculty, and patient views of barriers to cultural competence, and the skills needed to overcome them. Med Educ 2002;36:749-59.

20. Betancourt JR, Green AR, Carrillo JE, et al. Cultural competence and health care disparities: key perspectives and trends. Health Aff 2005;24:499-505.

21. Weech-Maldonado R, Elliott M, Pradhan R, et al. Can hospital cultural competency reduce disparities in patient experiences with care? Med Care 2012;50 Suppl-S48-S55.

22. Tervalon M, Murray-García J. Cultural humility versus cultural competence: a critical distinction in defining physician training outcomes in multicultural education. J Health Care Poor Underserved 1998;9:117-25.

23. Campinha-Bacote J. The Process of Cultural Competence in the Delivery of Healthcare Services: a model of care. $J$ Transcult Nurs 2002;13:181-4.

24. Richardson S, Williams T. Why is cultural safety essential in health care? Med Law 2007;26:699.

25. Carpenter-Song EA, Nordquest Schwallie M, Longhofer J. Cultural competence reexamined: critique and directions for the future. Psychiatr Serv 2007;58:1362-5.

26. Kumaș-Tan Z, Beagan B, Loppie C, et al. Measures of cultural competence: examining hidden assumptions. Acad Med 2007;82:548-57.
27. Braithwaite J, Hibbert P, Blakely B, et al. Health system frameworks and performance indicators in eight countries: A comparative international analysis. SAGE Open Med 2017;5:205031211668651.

28. Agency for Healthcare Research and Quality. Quality Improvement and monitoring at your fingertips. https://www.qualityindicators.ahrq. gov/ (accessed 01 Oct 2017).

29. Farquhar M. AHRQ quality indicators, 2008.

30. Donabedian A. Evaluating the Quality of Medical Care. Milbank Mem Fund Q 1966;44:166-206.

31. Arksey $H, O^{\prime}$ Malley L. Scoping studies: towards a methodological framework. Int J Soc Res Methodol 2005;8:19-32.

32. Levac D, Colquhoun H, O'Brien KK. Scoping studies: advancing the methodology. Implement Sci 2010;5:69.

33. Synthesis. Secondary Synthesis. 2016 http://synthesis.info/ (accessed 01 Oct 2017).

34. Jolley RJ, Lorenzetti DL, Manalili K, et al. Protocol for a scoping review study to identify and classify patient-centred quality indicators. BMJ Open 2017;7:e013632.

35. Donabedian A. The definition of quality and approaches to its assessment. Explorations in quality assessment and monitoring 1980.

36. Santana MJ, Manalili K, Jolley RJ, et al. How to practice personcentred care: A conceptual framework. Health Expect 2018.21.

37. World Health Organization. People-Centered Health Care: A Policy Framework Geneva: Switzerland: World Health Organization Western Pacific Region, 2007.

38. Weech-Maldonado R, Carle A, Weidmer B, et al. The Consumer Assessment of Healthcare Providers and Systems (CAHPS) cultural competence (CC) item set. Med Care 2012;50(9 Suppl 2):S22-.

39. Stewart AL, Nápoles-Springer AM, Gregorich SE, et al. Interpersonal processes of care survey: patient-reported measures for diverse groups. Health Serv Res 2007;42-1235-56.

40. Weech-Maldonado R, Dreachslin JL, Brown J, et al. Cultural competency assessment tool for hospitals: evaluating hospitals' adherence to the culturally and linguistically appropriate services standards. Health Care Manage Rev 2012;37:54-66.

41. Diversity in Action. A Toolkit for Residential Settings for Seniors, 2008.

42. Best Start Resource Centre. Giving Birth in a New Land: Strategies for Service Providers Working with Newcomers. Toronto: Best Start Resource Centre 2009.

43. The Lewin Group. Indicators of Cultural Competence in Health Care Delivery Organizations: An Organizational Cultural Competence Assessment Profile, 2002.

44. Strengthening Primary Care Access: Report of the Working Group to the Primary Healthcare Planning Group, 2011.

45. National Quality Forum. Measure Evaluation Criteria. http://www. qualityforum.org/Measuring_Performance/Submitting_Standards/ Measure_Evaluation_Criteria.aspx (accessed 01 Oct 2017).

46. Kongnetiman L, Eskow ED. Enhancing cultural competency: A resource kit for health care professionals: Calgary Health Region, 2009.

47. Champlain Valley Area Health Education Center. Cultural Competency For Health Care Providers, 2007.

48. Alberta Health Services. Diversity Awareness Self-Reflection Tool: Alberta Health Services, 2015.

49. Marshal S. A Cultural Competence Guide for Primary Health Care Professionals in Nova Scotia, 2005.

50. US Department of Health and Human Services. Cultural competence works: Using cultural competence to improve the quality of health care for diverse populations and add value to managed care arrangements. Washington, DC: Health Resources and Services Administration, DHHS, 2001.

51. Fongwa MN, Sayre MM, Ruth Anderson NL. Quality indicator themes among African Americans, Latinos, and Whites. J Nurs Care Qual 2008;23:50-7.

52. Lehman D, Fenza P. Hollinger-Smith L. Diversity \& Cultural Competency in Health Care Settings, 2016. 